APS, pDC and mDC produced BAFF and expressed chemokine receptors.

Conclusion $\mathrm{pDC}$ and $\mathrm{mDC}$ are differentially affected by IFN $\alpha$ in SLE and APS. IFN $\alpha$ primes pDC for enhanced IFN $\alpha$ production which potentiates $\mathrm{T}$-cell activation by $\mathrm{mDC}$, thereby sustaining the IFN signature in SLE and APS.

\section{PS5:92 CLARIFICATION OF THE ROLE OF DNASE 1 ON THE ONSET OF SYSTEMIC LUPUS ERYTHEMATOSUS IN A MURINE MODEL}

${ }^{1}$ E Kenny, ${ }^{2} \mathrm{U}$ Abu Abed, ${ }^{3} \mathrm{~A}$ Kuehl, ${ }^{1} \mathrm{~B}$ Raupach, ${ }^{2} \mathrm{~V}$ Brinkmann, ${ }^{1} \mathrm{~A}$ Zychlinsky. ${ }^{1}$ Max-PlanckInstitute for infection biology, dept. of Cellular Microbiology, Berlin, Germany; ${ }^{2}$ Max-PlanckInstitute for infection biology, Microscopy Core Facility, Berlin, Germany; ${ }^{3}$ Charite Universitaetsmedizin Berlin Forschungszentrum Immunwissenschaft RCIS, Berlin, Germany

10.1136/lupus-2018-abstract.137

Systemic lupus erythematosus (SLE) is a prototypical autoimmune disease resulting in multi-organ damage and a high rate of morbidity. Onset of SLE is characterised by dysregulated activation of $\mathrm{T}$ and $\mathrm{B}$ lymphocytes and the production of autoantibodies directed against nuclear components. The autoantibodies generated during the onset of SLE often recognise components released by neutrophils during NETosis, a type of cell death defined by the generation of neutrophil extracellular traps (NETs). The endonuclease DNase1 has been shown to be involved in the clearance of NET components. The sera of SLE patients contain inhibitors of DNase 1 and/or anti-NET antibodies that block the ability of DNase 1 to degrade NETs. Thus, whilst NETs are important for clearing infection they must be tightly regulated and degraded to prevent the onset of autoimmunity.

In this study we monitored the production of auto-antibodies in the serum of wild type and DNase 1-deficient mice from the age of 2 to 12 months, along with proteinuria levels and the development of glomerulonephritis. We show that DNase 1-deficient mice develop a SLE-like phenotype with elevated auto-antibody production and kidney damage by 12 months. This model also demonstrates the female bias in SLE as the female DNase 1-defieicnt mice had the highest level of kidney damage. As DNase I activity, B cells and aberrant NETosis are central to progression of SLE understanding their mechanisms of action are of great therapeutic interest.

\section{PS5:93 MARGINAL-ZONE-LIKE B CELLS DEFICIENCY REPEATEDLY DETECTED IN PERIPHERAL BLOOD AS A POSSIBLE BIOMARKER OF HYPOSPLENISM/ASPLENIA IN SLE}

${ }^{1} \mathrm{Z}$ Hrncir, ${ }^{2} \mathrm{D}$ Vokurkova, ${ }^{2} \mathrm{M}$ Drahosova, ${ }^{1} \mathrm{~T}$ Soukup, ${ }^{1} \mathrm{~J}$ Toms. ${ }^{1} /$ Ind Department of Internal Medicine, Charles University Hospital, Hradec Králové, Czech Republic; '2Department of Immunology and Allergy, Charles University Hospital, Hradec Králové, Czech Republic

\subsection{6/lupus-2018-abstract.138}

Objective SLE is a disease associated with a risk of serious infections, in case of hyposplenism/asplenia especially by encapsulated bacteria. For opsonization and phagocytosis of these agents are essential IgM natural Abs, produced only by B cells of the splenic marginal zone. Significant deficiency of marginal-zone-like B cell CD19 +CD27+IgM+ subpopulation absolute values $\mathrm{x} 10-6 / \mathrm{L}$ in peripheral blood $(\mathrm{PB})$ was demonstrated in a prospective, comparative, cross-over SLE study ${ }^{1}$; goal of the present study is follow up persistence of this phenomenon.

Design and method Sixty adult SLE (ACR/1982, update 1997) pts and 10 age-and sex-matched healthy controls (HC) were enrolled in month O', and 56 SLE pts also repeatedly after twelve-month-period, i. e. month 12'; overlap syndromes, infection, monoclonal gammopathy and renal failure in SLE under study were excluded. The DuraClone IM panel (Beckman Coulter) was used to identify CD19 +CD27+IgM+B cell subpopulation in $\mathrm{PB}$ samples by flow cytometry Navios (Beckman Coulter) with software analysis using Kaluza version 1.2: data obtained were expressed in relative $\%$ of PB lymphocytes and absolute values x10-6/L. Parallel analysis of serological SLE biomarkers included C3, C4, ANA/IF (maximal titre), ANA/ELISA, anti-dsDNA/IFCL (maximal titre), andi-dsDNA/ ELISA and antinucleosome Abs. Data obtained were statistically processed using Medcalc-Statistical Software programme.

Results Significant differences $(\mathrm{p}<0.001)$ were obtained between absolute values of CD19 $+\mathrm{CD} 27+\operatorname{IgM}+\mathrm{B}$ cells in HC (median 31.36, 95\% CI: 24.49 to 63.35 ) and SLE month O' (median 9.82, 95\% CI: 6.01 to 14.26), and also SLE month 12' (median 10.09 95\% CI: 7.12 to 14.42), but not between values obtained in SLE month $\mathrm{O}^{\prime}$ and month 12' ( $p>0.05)$; not significant differences were found in analysis using relative $\%$ of $\mathrm{PB}$ lymphocytes $(\mathrm{p}>0.05)$. In SLE month $\mathrm{O}$ ' was found a slight significant correlation between absolute values of CD19 $+\mathrm{CD} 27+\operatorname{IgM}+\mathrm{B}$ cells and anti-dsDNA/ELISA Abs ( $r s=-0.28, p=0.034$ ) without a confirmation in month 12' control ( $\mathrm{rs}=-0.09, \mathrm{p}=0.491$ ).

Conclusions The data obtained demonstrated persistent character of marginal-zone-like B cells deficiency in peripheral blood, and are suggesting as possible biomarker of functional hyposplenism/asplenia in SLE.

\section{REFERENCE}

1. Hrncir Z, et al. Clin Exper Rheumatol 2016;34(S99):S-63.

Acknowledgement Supported by the research project PROGRES Q40-15.

\section{PS5:94 CHARACTERISATION OF SLE B CELLS FROM PATIENTS IN REMISSION - PERSISTENT IL-10 SECRETORY DEFECT}

M Siekierka-Harreis, M Schroedter, R Brinks, B Opgenoorth, J Richter, S Vordenbäumen, M Schneider, G Pongratz. Rheumatology, Medical Faculty Heinrich-Heine University, Düsseldorf, Germany

\subsection{6/lupus-2018-abstract.139}

Background SLE is an autoimmune disorder characterised by polyclonal Bcell activation, the production of anti-double stranded (ds) DNA autoantibodies and cytokines. Molecular and clinical studies regarding SLE often address clinically active patientsand not patients in remission. This study reports on immunoglobulin, anti-dsDNA-aab and IL-10 secretory capacity of cultures of CD19 + lymphocytes from SLE patients in remission in comparison to normal donors. The aim was to evaluate whether endogenous factors (BAFF, CD40, IL4), exogenous factors (CpG-ODN-motifs, SAC) or their combinations differentially influence immunoglobulin, cytokine and anti-dsDNA-aab production in not active SLE patients vs healthy controls.

Methods Blood samples were obtained from a group of 13 SLE patients attending clinics at the rheumatology unit at the Heinrich- 
Heine University hospital in Düsseldorf and from 5 healthy controls (HC). All patients were randomly collected in clinical remission state (SLEDAI 1,1 $\pm 1,8$ ). The medication consisted of prednisolone (5/13 patients), mycophenolate mofetil (3/13 patients), azathioprine (1/13 patients), hydroxychloroquine (8/13 patients) or was without immunosuppression.

After 6 days of cell culture levels of $\operatorname{IgG}$, IgM, dsDNA antibodies and interleukin-10 (IL-10) were determined in the supernatants by ELISA. The effect of the stimuli alone or in combination on IgG, IgM, anti-dsDNA-aab, and IL-10 production was analysed.

Results Peripheral B cells from SLE patients in remission or control subjects did not show any difference in $\operatorname{IgG}, \operatorname{IgM}$, and anti-dsDNA-aabs to all aforementioned stimuli. The addition of $\mathrm{CpG}$ and SAC to cell cultures showed a stimulatory effect on immunoglobulin, cytokine and anti-dsDNA-aab production in SLE B cells and healthy controls alike. The amount of antidsDNA IgG-type autoantibodies produced by peripheral B cells was negligible. However, B cells from SLE patients showed diminished capacity to produce IL-10 as compared to B cells from healthy donors (SLE Estimate -40.17, Std.error 17.21, $\mathrm{p}<0.01$ ).

Conclusion B cells from SLE patients in remission as compared to peripheral B cells from healthy donors have comparable capacity to secrete immunoglobulin including non-IgG anti-dsDNA-aabs whereas their capacity to secrete IL-10 is impaired. This suggests a persisting intrinsic defect of $\mathrm{B}$ regulatory cells in SLE.

\section{PS5:95 CIRCULATING ANGIOGENIC T-CELLS ARE REDUCED IN PATIENTS WITH SYSTEMIC LUPUS ERYTHEMATOSUS WITH HIGH DISEASE ACTIVITY AND WITHOUT KNOWN CARDIOVASCULAR RISK FACTORS}

'S Piantoni, II Cavazzana, 'M Fredi, ${ }^{2} \mathrm{M}$ Taraborelli, ${ }^{1} \mathrm{~F}$ Franceschini, ${ }^{1} \mathrm{~A}$ Tincani, ${ }^{1} \mathrm{P}$ Airo'. ${ }^{1}$ Rheumatology and Clinical Immunology, Spedali Civili and University of Brescia, Italy; 2Internal Medicine Unit, Mellino Mellini Hospital, Chiari (Brescia), Chiari, Italy

\subsection{6/lupus-2018-abstract. 140}

Background Recent evidences underlined the central role of Tcells in the pathogenesis of Systemic Lupus Erythematosus (SLE) and in its cardiovascular complications. ${ }^{1}$ CD3 +CD31 +CXCR4+angiogenic T-cells (Tang) have been identified as a T-cell subtype involved in the repair of damaged endothelium cooperating with endothelial progenitor cells. ${ }^{2}$ Tang were described as selectively expanded in the circulation of systemic sclerosis patients displaying peripheral vascular complications, as a reaction to an inefficient angiogenesis. ${ }^{3}$ Not much information is available on Tang in a SLE patients: in a recent study the percentage of circulating CD8 + Tang, but not CD4 + Tang, was higher in SLE than in healthy controls. ${ }^{4}$ However, in this study SLE patients with hypertension, dyslipidemia or smoking habit, factors which may influence Tang counts, were not excluded.

The aim of this study was to characterise Tang in a cohort of patients with SLE without known cardiovascular risk factors.

Methods Twenty female SLE patients with a recent disease onset $(<5$ years) and without traditional cardiovascular risk factors or previous events (age: median value $=43[25$ th- 75 th percentile $=27-54]$ years) and 18 healthy controls (age: 40 [32-54] years) were enrolled.

Phenotypic analysis of peripheral Tang lymphocytes was made by flow-cytometry.

Disease activity was evaluated by SLEDAI-2K score.

Results SLE patients were divided in two groups according with disease activity. Patients with SLEDAI-2K equal or higher than 6 were defined as patients with high disease activity (n:5). They had a lower percentage of circulating Tang in comparison with healthy controls $(10$ [8-15] vs 16 [14-23]\% of CD3 + T cells, $\mathrm{p}=0.04)$. The result was confirmed in absolute number (83 [60-103] vs 242 [165-328] cell/microliter, $\mathrm{p}=0.04)$. SLE patients with low disease activity had levels of Tang which were intermediate between, and not significantly different from, healthy controls and patients with high disease activity.

Conclusions Tang were reduced in our patients with active SLE, and no known cardiovascular risk factors, suggesting that this reduction was directly explained by disease activity.

\section{REFERENCES}

1. Mak A. J Immunol Res 2014

2. Hur J. Circulation 2007.

3. Manetti M. PLoS One 2017.

4. Miao J. Mediators Inflamm 2016.

\section{PS5:96 IL-21 DEPENDENT GRANZYME B PRODUCTION OF B-CELLS IS DECREASED IN PATIENTS WITH LUPUS NEPHRITIS}

${ }^{1} \mathrm{M}$ Rabani, ${ }^{1} \mathrm{~B}$ Wilde, ${ }^{1} \mathrm{~K}$ Hübbers, ${ }^{1} \mathrm{~A}$ Kribben, ${ }^{2} \mathrm{O}$ Witzke, ${ }^{2} \mathrm{~S}$ Dolff. ${ }^{1}$ Department of Nephrology, University Hospital Essen, Germany; ${ }^{2}$ Department of Infectious Diseases, University Hospital Essen, Germany

\subsection{6/lupus-2018-abstract. 141}

Objectives B-cells play a crucial role in the pathogenesis of lupus nephritis. Recently, a separate subset has been discovered characterised by expression of Granzyme B. The aim of this study is to investigate this subset in patients with systemic lupus erythematosus especially in patients with lupus nephritis. Methods Isolated peripheral blood mononuclear cells of patients with systemic lupus erythematosus $(n=30)$ and healthy controls $(\mathrm{n}=21)$ were in vitro stimulated with $\mathrm{CPG}$, $\operatorname{IgG}+\operatorname{IgM}$ and IL-21. Patients were sub-grouped in patients with and without biopsy proven lupus nephritis. CD19 + B cells were analysed for intracellular Granzyme B expression by flow cytometry. Patients disease activity was assessed by systemic lupus erythematosus disease activity index (SLEDAI).

Results The strongest stimulus for Granzyme B secretion of CD19 + B cells was IgG $+\operatorname{IgM}$ in presence of IL-21. Patients with systemic lupus erythematosus had a significant decreased percentage of Granzyme B $+\mathrm{CD} 19+\mathrm{B}$ cells. This could be shown in particular for patients with active disease and with lupus nephritis.

Conclusions These data demonstrate that CD19 + B cells of patients with systemic lupus erythematosus are impaired to produce Granzyme B. This may contribute to an imbalanced B-cell regulation towards effector B-cells which might promote the development of lupus nephritis. 